\title{
Riscos ergonômicos e biomecânicos ocupacionais no transporte de pacientes no centro cirúrgico: pesquisa qualiquantitativa de estudo transversal
}

\section{Occupational ergonomic and biomechanical risks in patient transporting in the operating room: Qualitive and Quantitative research of cross-sectional study}

\author{
Vanessa Silva de Quevedo', Arlete Ana Motter², Rubia Bayerl'3, \\ Flávia Cristina Miranda4, Paula Chomem5 ${ }^{5}$, Angela Luiza Cunha Legey ${ }^{6}$
}

'Autora para correspondência. Universidade Federal do Paraná. Curitiba, Paraná, Brasil. ORCID: 0000-0003-2716-3670. vanessa.quevedoo1997@gmail.com 2Universidade Federal do Paraná. Curitiba, Paraná, Brasil. ORCID: 0000-0002-2585-207X. arlete.motter@uol.com.br ${ }^{3}$ Universidade Federal do Paraná. Curitiba, Paraná, Brasil. ORCID: 0000-0001-9004-3490. rubiabayerlufpr@gmail.com ${ }^{4}$ Universidade Federal do Paraná. Curitiba, Paraná, Brasil. ORCID: 0000-0002-9996-0874. flacmiranda@gmail.com 5Universidade Federal do Paraná. Curitiba, Paraná, Brasil. ORCID: 0000-0003-4298-6237. paulachomem@gmail.com ${ }^{6}$ Universidade Federal do Paraná. Curitiba, Paraná, Brasil. ORCID: 0000-0002-4295-4182. angelalegey@gmail.com

RESUMO | INTRODUÇÃO: OBJETIVO: Investigar os riscos ergonômicos e biomecânicos ocupacionais em profissionais da enfermagem no transporte de pacientes, no centro cirúrgico de um hospital público. METODOLOGIA: Estudo de caráter exploratório, observacional e descritivo. Para tanto, utilizou-se o Questionário Internacional da Atividade Física (IPAQ), que analisa diferentes contextos do cotidiano para estimar o tempo semanal gasto em caminhadas, atividades físicas de intensidade moderada, vigorosa e atividades passivas (tempo sentado). O Questionário Nórdico, possibilita a identificação de distúrbios osteomusculares nos 12 meses e 7 dias anteriores à entrevista. Permite também um diagnóstico do posto de trabalho pela sua relação com a prevalência do local e tempo do surgimento dos sintomas dolorosos. Além disso, aplicou-se a metodologia da Análise Ergonômica do Trabalho (AET). RESULTADOS: De acordo com a análise dos dados coletados participaram deste estudo 44 profissionais da enfermagem, $32(72,7 \%)$ eram do gênero feminino e $13(29,54 \%)$ do gênero masculino. Quando analisado a especialidade: 24 (54,5\%) eram auxiliares de enfermagem, técnicos de enfermagem correspondem à $14(31,8 \%)$ e Enfermeiros $6(13,6 \%)$. O questionário IPAQ demonstrou que 16 (35\%), destes profissionais mantém-se ativos. Por meio da análise ergonômica do trabalho, as variáveis como, tipo de maca, condições de manutenção, tipo de anestesia, idade do paciente, entre outros, interferem em maiores ou menores esforços no transporte de pacientes. CONCLUSÃo: Desse modo, tornam-se necessárias a adoção de medidas preventivas que visem a melhoria do estado de saúde prevenindo complicações musculoesqueléticas no desempenho das atividades. Além de promover boas condições de trabalho aos profissionais no seu ambiente profissional, bem como trazer benefícios ao hospital.
ABSTRACT | INTRODUCTION: OBJECTIVE: To investigate occupational ergonomic and biomechanical risks in nursing professionals in patient transporting in the operating room of a public hospital. METHODOLOGY: Exploratory, observational and descriptive study. For this the International Physical Activity Questionnaire (IPAQ) was used, which analyzes different daily contexts to estimate weekly time spent walking, moderate, vigorous intensity physical activity and passive activities (sitting time). The Nordic Questionnaire enables the identification of musculoskeletal disorders in the period of 12 months and 7 days prior to the interview. It also allows a diagnosis of the workplace by its relation with the prevalence of the place and time of the onset of painful symptoms. In addition, the methodology of Ergonomic Workplace Analysis (EWA) was applied. RESULTS: According to the analysis of the collected data, 44 nursing professionals participated of this study, $32(72.7 \%)$ were female and $13(29.54 \%)$ were male. When analyzing the specialty: $24(54.5 \%)$ were nursing assistants, nursing technicians correspond to $14(31.8 \%)$ and Nurses $6(13.6 \%)$. The IPAQ questionnaire showed that 16 (35\%) of these professionals remain active. Through ergonomic analysis of the workplace, variables such as stretcher type, maintenance conditions, type of anesthesia, patient age, among others, interfere with greater or lesser efforts in patient transport. CONCLUSION: Thus, it becomes necessary the adoption of preventive measures aimed at improving health by preventing musculoskeletal complications in the performance of activities. Besides promoting good working conditions for professionals in their professional environment, as well as bringing benefits to the hospital.

KEYWORDS: Ergonomic analysis. Nursing. Operating room. Occupational risks. 


\section{Introdução}

O trabalho é uma ferramenta essencial para o ser humano, visto que permite a formação de uma identidade profissional dentro de um contexto social. No entanto o trabalho está sujeito a diferentes situações que podem se relacionar à própria profissão ou a forma com que este é estruturado e se desenvolve, podendo ocasionar distúrbios osteomusculares aos trabalhadores inclusive na área da saúde, mais especificamente nos profissionais da enfermagem².

Os distúrbios osteomusculares, frequentemente observados no profissional da enfermagem atuante no centro cirúrgico são relacionados à coluna vertebral, com maior prevalência na região cervical e lombar, e que se caracterizam pela presença de dor, parestesia, sensação de peso, fadiga e restrição na realização de algum movimento ${ }^{4}$. Geralmente acontecem os membros superiores, mas podem acometer membros inferiores resultando em incapacidade para a realização do trabalho, e pode haver sobrecarga das estruturas anatômicas do sistema osteomuscular, relacionada à falta de tempo para recuperação, movimentos repetitivos, com ou sem esforço localizado ou adoção de determinadas posições por tempo prolongado ${ }^{2,3}$.

Estas condições quando relacionadas aos fatores químicos, biológicos, físicos e ergonômicos podem causar consequências à saúde do trabalhador na área da enfermagem, constantes riscos encontrados em um centro cirúrgico ${ }^{5}$.

Dentre estes fatores, pretende-se elucidar neste estudo os riscos ergonômicos e os riscos biomecânicos ocupacionais que são preditores absolutos para o surgimento dos distúrbios musculoesqueléticos. Um risco ergonômico pode ser definido como uma condição ou uma prática que traga obstáculos à produtividade, que desafie a boa qualidade ou que traga prejuízos ao conforto, segurança e bem-estar do trabalhador6. Já o risco biomecânico ocupacional pode ser definido como, posturas inadequadas adotadas durante a jornada de trabalho juntamente com o manuseio de cargas que ocasionam tensões osteomusculares.
As cargas mais leves aplicadas continuamente podem provocar inflamação, devido ao estiramento prolongado dos tecidos das estruturas solicitadas no movimento. Por sua vez, cargas pesadas podem alterar os tecidos quando ultrapassam a capacidade física de resposta, produzindo lesão muscular ou ligamentar? .

As Lesões por Esforços Repetitivos ou Distúrbios Osteomusculares Relacionados ao Trabalho (|LER/ DORT) são decorrentes de movimentos repetidos, entre outros fatores, de qualquer parte do corpo que podem ocasionar lesões acompanhadas de dor, em resposta à inflamação ou degeneração de tendões, nervos, ligamentos, músculos e estruturas periarticulares em diferentes estruturas anatômicas ${ }^{8}$. Em comum, esses termos designam inflamações localizadas, síndromes nervosas compressivas ou síndromes dolorosas?.

Além disso, o rápido desenvolvimento da tecnologia em saúde e o crescimento de novos procedimentos e exames, fazem com que, o conhecimento exigido na área da saúde seja grande. Ainda, deve-se considerar o ritmo de trabalho, o contato do profissional com o paciente, com a dor e com a morte, elementos que contribuem para os riscos a saúde física, mental e biológica do profissional da enfermagem. Sendo assim, o centro cirúrgico é considerado como cenário de alto risco, onde os processos de trabalho constituem-se em práticas complexas, interdisciplinares, com forte dependência da atuação individual e da equipe em condições ambientais dominadas por pressão e estresse ${ }^{10}$.

A equipe de enfermagem é imprescindível para a excelência do funcionamento e gerenciamento de toda a equipe médica. Em um centro cirúrgico os profissionais da enfermagem desempenham diversas funções dentro do ambiente de trabalho, além de empenhar-se por diversas horas ou ainda dedicar-se ao período de contraturno à segundo emprego, como forma de complementar a renda ou buscar maior sucesso e valorização profissional. A Associação Americana de Enfermagem, define a profissão de enfermagem como a proteção, promoção e otimização da saúde, sendo responsabilidade intrínseca do profissional de enfermagem atuar na prevenção de doenças e 
lesões, na facilitação da cura, no alívio do sofrimento por meio do diagnóstico e tratamento do ser humano, e no atendimento de indivíduos, famílias, grupos, comunidades e populações em geral ${ }^{5}$.

Outrossim em exercício diário, os profissionais da enfermagem são responsáveis pela movimentação e deslocamento de pacientes. Diante disso, esses trabaIhadores permanecem por longos períodos em pé e trabalham com equipamentos obsoletos do ponto de vista ergonômico, e ainda estão submetidos a terem poucas horas de sono e descanso. Tais condições contribuem para a ocorrência dos riscos biomecânicos e ocupacionais, os quais resultam em elevados pedidos de afastamentos, licenças e aposentadorias por invalidez devido à sobrecarga, em decorrência disso, há uma potencialização das desordens musculoesqueléticos ${ }^{11}$.

Neste sentido, a ergonomia tem como objetivo teórico conhecer e compreender a forma com que o trabalho humano se desenvolve, informando sempre o profissional a respeito de sua carga de trabalho de acordo com a atividade em particular de cada trabalhador. Ademais como objetivo prático, em primeiro lugar visa o bem-estar do trabalhador, como saúde, segurança e o bom relacionamento com o ambiente de trabalho ${ }^{12}$.

Assim, o objetivo do estudo foi investigar os riscos ergonômicos e biomecânicos ocupacionais em profissionais da enfermagem no transporte de pacientes no centro cirúrgico, levando em consideração os distúrbios mais frequentemente encontrados no profissional da enfermagem.

\section{Metodologia}

Trata-se de um estudo exploratório, observacional e descritivo, com análise de resultados qualitativo e quantitativo. A pesquisa foi aprovada pelo Comitê de Ética em Pesquisa (CEP) do Setor de Ciência da Saúde da UFPR em 12 de julho de 2017, sob o número: 2015017272.
O estudo foi norteado pela Análise Ergonômica do Trabalho AET. Que orienta ações relacionadas ao levantamento de peso, transporte e descarga de materiais, condições ambientais e a organização do trabalho ${ }^{13}$.

As variáveis numéricas foram exploradas pela média, mediana e moda; mínima, máxima, desvio padrão; frequências absolutas e percentuais. Todas as análises foram realizadas utilizando o software livre de computação estatística $R$, na versão 3.5.3. Os dados foram organizados em tabelas. As associações entre as variáveis estudadas, foram analisadas a partir do coeficiente de correlação de Pearson, que mede o grau da correlação linear entre duas variáveis quantitativas e/ou variáveis categorizadas, sendo que este coeficiente varia entre -1 e 1. Os valores negativos, demonstram uma associação inversa entre as variáveis, já os valores positivos uma associação linear. Quanto mais próximo de 1 , a correlação é considerada forte, e quando os valores mais próximos de 0 , é considerada fraca.

O estudo foi realizado na Unidade do Centro Cirúrgico (UCC) de um hospital universitário do sul do país entre janeiro a dezembro de 2018. Neste local ocorre o atendimento à pacientes adultos e pediátricos em caráter eletivo, de urgência ou emergência e cirúrgica.

Participaram da pesquisa 44 profissionais da equipe de enfermagem (de um total de 64 ), os quais assinaram previamente o Termo de Consentimento Livre e Esclarecido (TCLE). A identificação, triagem e elegibilidade e inclusão seguiu o fluxograma da Figura 1. Os participantes receberam os questionários, Nórdico e IPAQ (autoaplicáveis) foram observados durante a execução das suas atividades. Os resultados do estudo foram apresentados aos participantes ao final da pesquisa em uma reunião, com a presença da chefia. 

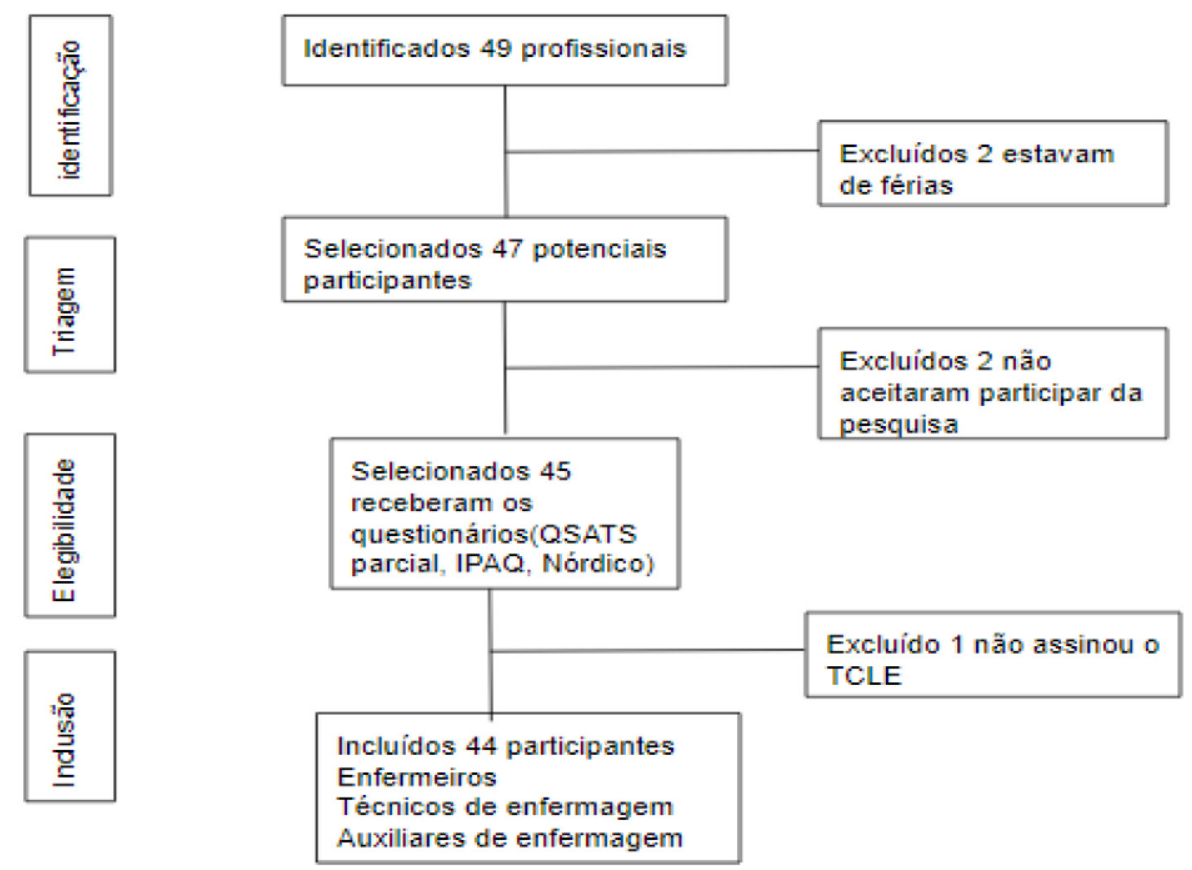

A metodologia baseou-se na análise Ergonômica do Trabalho (AET) através das etapas análise da demanda, da tarefa e da atividade ${ }^{13}$. A análise da demanda permite delimitar o problema a ser abordado, é o que permite por fim, traçar um plano de intervenção. Já a análise da tarefa compreende a investigação do trabalho como um todo, identificando as diferenças entre o trabalho prescrito e o real. A análise da atividade, por sua vez, através da observação do trabalhador individualmente, identifica os fatores próprios do indivíduo e externos relacionados ao ambiente e as condições de trabalho, que o influenciam no processo laboral ${ }^{12}$.

Na etapa quantitativa avaliou-se o profissional no período pós-operatório, pois foi identificado, como o maior gerador de esforços físicos. As observações incluíram dois momentos: transporte do paciente da sala cirúrgica até a sala de recuperação pós anestésicos ou UTI e transporte do paciente dessa sala para a enfermaria. As variáveis observadas incluíram, o tipo de anestesia utilizada, a idade do paciente, estatura, tipo de maca, entre outros.

Para a análise quantitativa, incluiu, o Questionário Internacional da Atividade Física (IPAQ-versão curta) e o Questionário Nórdico, que utiliza diferentes contextos do cotidiano para estimar o tempo semanal gasto em caminhadas, atividades físicas de intensidade moderada e vigorosa e atividades passivas (tempo sentado). A versão curta é constituída por sete questões abertas e suas informações permitem analisar o nível de atividade física em uma semana. A partir das respostas do participante, pode-se classificá-lo em muito ativo, ativo, irregularmente ativo $A$, irregularmente ativo $B$ e sedentário, conforme a frequência e a duração das atividades vigorosas, moderadas e caminhadas ${ }^{14}$.

O indivíduo classificado como muito ativo pratica atividades vigorosas em cinco dias da semana por pelo menos 30 minutos ou as realiza três vezes na semana por 20 minutos acompanhados de 30 min de atividades moderadas ou caminhadas. O participante ativo foi considerado aquele que pratica 20 minutos de atividade vigorosa em três dias da semana, ou 30 minutos de atividades moderadas ou caminhada em cinco dias da semana, ou ainda aquele que pratica 150 minutos de atividade contabilizando as vigorosas, moderadas e caminhadas. 
O questionário Nórdico visão geral é um instrumento que possibilita a identificação de distúrbios osteomusculares nos 12 meses e 7 dias anteriores à entrevista. Dessa forma, permite também um diagnóstico do posto de trabalho pela sua relação com a prevalência do local e tempo do surgimento dos sintomas. O questionário contempla as regiões do pescoço, ombros, parte superior das costas, cotovelo, punhos e mãos, parte inferior das costas, quadril e coxa, joelhos, tornozelos e pés abordando questões sobre a presença de dor, formigamento e dormência nos últimos 12 meses; limitação para realizar atividades de vida diária e a consulta em algum profissional da saúde.

\section{Resultados}

Para a descrição dos resultados, os dados quantitativos foram expressos por meio da estatística descritiva, a partir da média, desvio padrão e frequência. Com base nos resultados qualitativos da investigação foi realizado o diagnóstico e foram propostas as recomendações ergonômicas apresentadas em uma última reunião com a finalidade de oferecer um feedback aos profissionais. Os dados qualitativos foram baseados na análise do trabaIho do profissional da enfermagem deste centro cirúrgico, buscou-se verificar se o trabalho real possui alguma relação com os resultados apresentados nos questionários utilizados nesta pesquisa.

Participaram 44 profissionais de enfermagem do centro cirúrgico analisado, sendo os profissionais: auxiliares de enfermagem 24 (54,5\%), técnicos de enfermagem 14 (31,8 \%) e enfermeiros 6 (13,6\%), desses, 32 (72,7\%) eram do gênero feminino e 13 (29,54\%) do gênero masculino. A idade média foi de 42.3 anos, sendo a idade mínima 26 anos e a máxima 75 anos. $O$ tempo médio na função era de 16 anos, variando de 2 meses a 55 anos.

O centro cirúrgico analisado é considerado de grande porte, são 14 salas cirúrgicas, em média 35 cirurgias por dia. A enfermagem passa o paciente ainda sob efeito anestésico, da mesa de cirurgia para a maca. Durante a jornada de trabalho, os profissionais fazem longas caminhadas e permanecem muito tempo em pé.

Figura II. Caracterização dos participantes da pesquisa

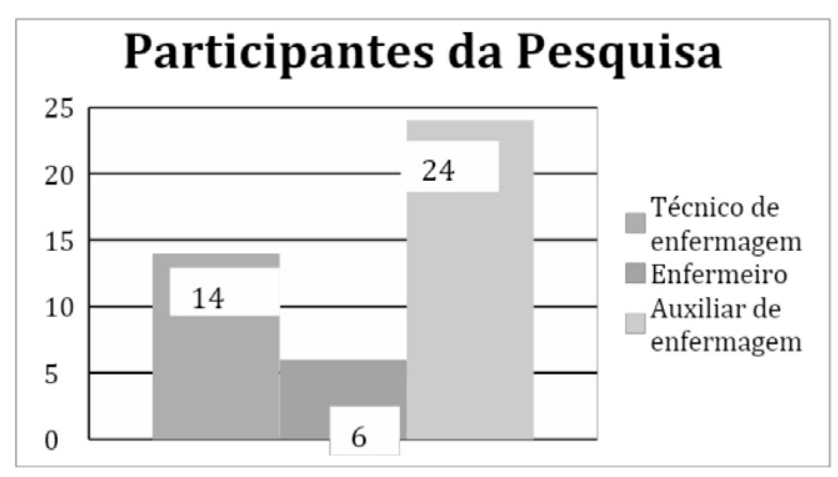

Fonte: os autores.

Os resultados do IPAQ demonstraram que 6 (13\%) eram sedentário, 12 (28\%) irregularmente ativo, regularmente ativos $4(8 \%)$, ativo $16(35 \%)$, muito ativo $6(13 \%)$. 


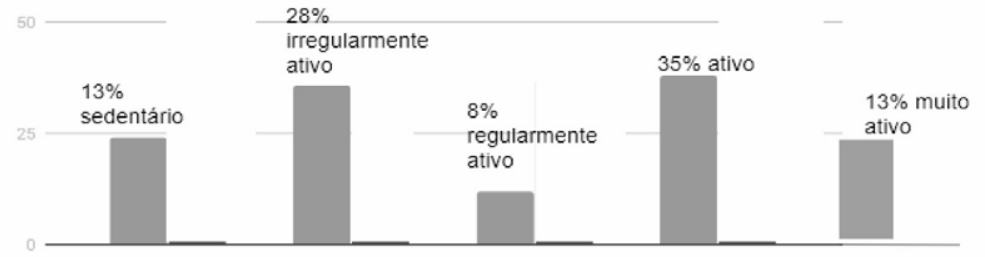

Fonte: os autores.

O questionário Nórdico demonstrou que a região mais acometida por dor, era a região lombar, seguida por ombro, tórax, joelhos e tornozelos.

Figura IV. Resultados do questionário Nórdico

\begin{tabular}{|c|c|c|c|c|}
\hline Estruturas & $\begin{array}{l}\text { Nos últimos } 12 \\
\text { meses teve } \\
\text { problemas em } \\
n(\%)\end{array}$ & $\begin{array}{l}\text { Foi impedido de } \\
\text { realizar atividades } \\
\text { normais } \mathrm{n}(\%)\end{array}$ & $\begin{array}{l}\text { Consultou } \\
\text { profissional da } \\
\text { saúde } \\
\mathrm{n}(\%)\end{array}$ & $\begin{array}{l}\text { Nos últimos } 7 \text { dias } \\
\text { n (\%) }\end{array}$ \\
\hline & $15(31,81 \%)$ & $4(9,09 \%)$ & $8(18,18 \%)$ & $6(13,63 \%)$ \\
\hline Ombro & $17(34,09 \%)$ & $3(6,81 \%)$ & $5(11,36 \%)$ & $7(15,90 \%)$ \\
\hline$x$ & $17(38,63 \%)$ & $8(18,18 \%)$ & $5(11,36 \%)$ & $10(22,72 \%)$ \\
\hline Cotovelo & $5(11,36 \%)$ & $4(9,09 \%)$ & $4(9,09 \%)$ & $1(2,27 \%)$ \\
\hline & $14(31,81 \%)$ & $8(18,18 \%)$ & $6(13,63 \%)$ & $2(4,54 \%)$ \\
\hline Lombar & $23(52,27 \%)$ & $9(20,45 \%)$ & $9(20,45 \%)$ & $15(31,81 \%)$ \\
\hline Quadril/coxas & $10(22,72 \%)$ & $2(4,54 \%)$ & $4(9,09 \%)$ & $2(4,54 \%)$ \\
\hline Joelhos & $15(34,09 \%)$ & $6(13,63 \%)$ & $9(20,45 \%)$ & $9(20,45 \%)$ \\
\hline Tornozelos/pés & $16(38,63 \%)$ & $6(13,63 \%)$ & $7(15,90 \%)$ & $7(15,90 \%)$ \\
\hline
\end{tabular}

De acordo com a análise estatística foi possível estabelecer uma relação positiva em conformidade com a variável de dor no pescoço, conforme o tempo na função $(0.22$ p-valor $=0.14)$, não apresentou relação direta com o sexo (0.03 p-valor $=0.83)$ e idade $(0.66 \mathrm{p}$-valor $=0.00)$, ou seja, o nível da dor foi presente invariavelmente no sexo feminino e masculino. Com relação à dor no ombro se demonstrou positiva quando relacionada à idade $(p=0.14)$ e tempo na função dos participantes ( $p=0.06)$. 
A presença de dor na região superior das costas não apresentou relação com o sexo e nem sequer com o tempo na função, mas sim com a idade, ou seja, maior a probabilidade estatística para a ocorrência de dor na região superior das costas em indivíduos com idade mais avançada. Apresentou correlação positiva a presença de dor nas mãos e nos punhos quando relacionada ao sexo feminino, idade e foi negativa com relação ao tempo na função. Nos membros inferiores a dor obteve correlação com a idade e o sexo feminino. A dor nos tornozelos e pés foi positiva somente quando correlacionada com o sexo feminino sendo negativa para idade e tempo na função, dados apresentados na figura $\mathrm{V}$.

Ainda na verificação de correlação das respostas demonstrada na figura $V$, o item dormir em horários pouco usuais com o turno de trabalho verificou-se uma correlação chegando a ser moderada e negativa. Ou seja, as pessoas que possuem uma jornada diurna, não se sentem incomodadas com o sono, o que era esperado. Foram analisadas outras variáveis como demonstrado abaixo (figura V).

Figura V. Correlações com o questionário Nórdico, contendo p-valor do teste de correlação de Pearson

\begin{tabular}{|l|l|l|l|l|}
\hline Variável & Idade (p-valor) & Sexo (p-valor) & $\begin{array}{l}\text { Tempo na função (p- } \\
\text { valor) }\end{array}$ & $\begin{array}{l}\text { Incomodados com o sono } \\
\text { (p-valor) }\end{array}$ \\
\hline Dor no pescoço & $0.66(<0.001)$ & $-0.06(0.64)$ & $0.22(0.14)$ & - \\
\hline Dor na região superior das costas & $0.02(0.88)$ & $-0.02(0.86)$ & $-0.14(0.34)$ & - \\
\hline Dor no punho e nas mãos & $0.06(0.64)$ & $0.03(0.79)$ & $-0.02(0.89)$ & - \\
\hline Dor nos MMII & $0.05(0.69)$ & $0.09(0.54)$ & $-0.07(0.86)$ & - \\
\hline Dor nos tornozelos e pés & $-0.05(0.69)$ & $0.10(0.48)$ & $-0.09(0.54)$ & $-0.41(0.94)$ \\
\hline Dormir em horário pouco usuais & - & - & - & \\
\hline Fonte: os autores & & & & \\
\hline
\end{tabular}

O trabalho da enfermagem do centro cirúrgico muitas vezes ultrapassou o trabalho prescrito. Ocorrem cirurgias emergenciais que geram alteração de toda a rotina do trabalho e a necessidade de se implementar estratégias que não prejudiquem os pacientes; também se observou a precária manutenção das macas, por falta de profissionais que realizam o serviço, fazendo com que a própria equipe de enfermagem realize os reparos, condição que o predispõe a riscos, já que utilizam posturas e materiais inadequados para recorrer às improvisações. Quanto aos equipamentos empregados no hospital, surge uma preocupação, uma vez que estes devem ser seguros e com manutenção periódica. Torna-se indispensável avaliar a escassez dos equipamentos para o transporte, principalmente de macas, com o intuito de evitar a sua ausência ou falhas no funcionamento durante o transporte dos mesmos.

Durante as observações realizadas durante a coleta de dados no centro cirúrgico, alguns profissionais relataram algumas queixas álgicas em decorrência do trabalho e que são frequentes, o que se relaciona diretamente comas respostas obtidas no questionário nórdico. Os questionamentos foram obtidos durante o pós-operatório, momento este em que o paciente ainda se encontra sob efeito anestésico e por isso facilitou mais a interação dos pesquisadores com os profissionais. A seguir alguns breves relatos dos participantes que estão identificados em ordem numérica, (P1, P2, P3 e assim sucessivamente):

Sexo feminino 30, "Tenho dores fortes na lombar e cervical" (P1).

Sexo masculino 43, "Sinto dor em trapézio e nas costas" (P2).

Sexo masculino 25, "Dor no braço esquerdo, mas acho que foi por que eu cai vindo até aqui de bicicleta" (P3). 
Sexo feminino 40, "Tenho muita dor de cabeça e no trapézio" (P4).
"Fico sentada no chão para realizar a limpeza das rodas, ao final do dia estou com uma imensa dor nas costas" (P2).

A AET também permitiu constatar outros fatores que podem impactar nos esforços físicos realizados pelos profissionais de enfermagem: a) o tipo de anestesia utilizada, o que acarretará no seu efeito ou tempo de reação ao fármaco, mais tardio ou mais rápido e além disso, na extensão do efeito anestésico no corpo do paciente (por exemplo, o paciente com anestesia local poderá ajudar nas transferência, enquanto que aquele que fez uma anestesia raquidiana contribuirá menos); b) tipo de procedimento cirúrgico, pois de acordo com a extensão da cirurgia, possibilitará em maior ou menor movimentação do paciente; c) idade do paciente, pois observou-se que quando o paciente era mais idoso ou criança respondiam menos aos comandos de voz da enfermagem no momento do transporte; d) o peso e a estatura, sendo que o paciente de maior peso e estatura resultaram em maior esforço realizado pelo profissional no momento do transporte; e) a falta de manutenção das macas; f) o tipo de maca, pois as macas mais antigas são mais pesadas para o profissional do transporte, entretanto, estão disponíveis em maior número neste centro cirúrgico, essa observação se afirma ao relato de um dos profissionais do transporte:

"Essa maca da enfermaria é muito melhor de carregar, notei que o paciente se sente mais seguro também, já que não sente tanta crepitação" (P4), sua colega então responde: "Nossa eu prefiro também, além de ser confortável ao paciente é bem mais leve para carregar"(P5).

do médico Ele falou que é desgaste da profissão em si e trabalhar a vida toda puxando, força excedente" (P6).

A falta de manutenção das macas, entre outros fatores, pode acarretar maior sobrecarga ao profissional da enfermagem que realizará o transporte do paciente além dos possíveis riscos biomecânicos e ocupacionais pela maior geração de esforços e dispêndio energético. Por exemplo, na sala de recuperação pós anestésicos, um técnico de enfermagem realizava as limpezas das macas que estavam com problemas para o transporte e que não tinham previsão para uma manutenção especializada. Assim, retirava os excessos de fios e dejetos depositados nas rodas da maca com uma tesoura ou uma caneta, correndo risco de lesão com perfurocortantes. O relato a seguir ilustra a dificuldade dessa situação, que repercute em queixas de dores musculoesqueléticas:

\section{Discussão}

Nesse estudo também se verificou um maior contingente de profissionais do sexo feminino, condizente com estudo sobre o perfil da enfermagem brasileira realizado pelo Conselho Federal de Enfermagem (COFEN), no qual se evidenciou a predominância de enfermeiras na categoria profissional $(84,6 \%)^{15}$. Para Nauderer \& Lima Mads20, o contexto histórico do cuidado atrelado à figura feminina projetou alguns estereótipos sobre o cuidar da enfermagem que foram enraizados no imaginário social através dos tempos. 
Com relação às condições de trabalho e aos grandes esforços adotados pelos profissionais que participaram deste estudo, nota-se uma sobrecarga associada ao trabalho da enfermagem, por falta de meios técnicos que possam amparar as questões relacionadas a manutenção de objetos e equipamentos que são úteis e imprescindíveis ao próprio exercício profissional.

O sistema de saúde é resultante de um complexo sistema sócio técnico, tornando-o vulnerável às condições de insegurança nas quais o profissional da enfermagem se torna suscetível ${ }^{13}$, observando-se como resultante, que a segurança do paciente pode ficar comprometida, assim como encontrado em um estudo feito por Gonçalves \& Andolhe ${ }^{21}$ em uma Unidade de Terapia Intensiva (UTI), quando existe maior carga de trabalho, e por conseguinte, maior frequência de eventos adversos no setor.

Os cuidados com as condições de trabalho (incluindo carga horária, carga de trabalho e disponibilidade de recursos) estão relacionadas com a melhora desempenho de funções deste profissional o que consequentemente resulta em maior segurança no cuidado. Para propiciar tudo isso, faz-se necessário que os gestores compreendam que a promoção de melhores condições de trabalho se relaciona intimamente com a qualificação da assistência e que, ao negligenciar este aspecto, a sua conduta será contraproducente com os princípios da gestão para a qualidade ${ }^{19}$.

Compete aos gestores rever os processos de trabaIho e reforçar as linhas de comunicação, pois, apenas desta forma será possível o estabelecimento de estratégias que permitam impedir falhas que estão relacionadas ao cuidado e que tornam o sistema frágil.

Em relação às queixas relacionadas à manutenção de equipamentos no trabalho, estudos revelam que a falta de estrutura física adequada e recursos humanos acarreta a sobrecarga de trabalho, submetendo o funcionário a fortes imposições e situações imprevisíveis, causando cansaço físico e emocional ${ }^{6}$.

No Brasil, cerca de $60 \%$ dos brasileiros não praticam nenhum tipo de atividade física observado em um estudo realizado em uma UBS (Unidade Básica de Saúde) da cidade de Floriano, Piauí, Brasil, que verificaram a prevalência de comportamento sedentário em enfermeiros, técnicos de enfermagem e agentes comunitários de saúde. No estudo citado, foram identificados que $55,6 \%$ dos profissionais participantes da pesquisa são considerados sedentários².

Com relação a presença de dor pelo questionário Nórdico obtivemos presença de dor em pescoço, coluna, mãos, punhos, membros inferiores, pé e tornozelos. Quanto à dor no tornozelo e em membros inferiores, pode-se inferir que está relacionada a fatores individuais, fatores relacionados ao trabalho, como carga horária e aspecto de cada setor, são relevantes para o surgimento de complicações, que acaba por refletir em quadros álgicos na região da coluna, assim como proposto no estudo de Reed LF, Battistutta $\mathrm{D}$, Young J \& Newman $\mathrm{B}^{18}$.

Uma pesquisa feita com 29 profissionais de enfermagem, sendo três auxiliares, 23 técnicos e três enfermeiros, se observou a prevalência de dores musculoesqueléticas $(96,6 \%)$ em pelo menos uma das 17 regiões corporais nos últimos 12 meses. As principais regiões anatômicas acometidas pelos profissionais analisados foram as partes inferiores e superiores das costas (79,3 e $75,9 \%$, respectivamente), o pescoço $(65,5 \%)$, os ombros $(62,1 \%)$, os tornozelos/pés $(55,2 \%)$ e punhos/ mãos (51,7\%). Além disso, acresce que $65,5 \%$ dos investigados relataram ter se afastado do trabalho por motivos de saúde nos últimos 12 meses $^{2}$.

Esses resultados divergem dos achados desta pesquisa sendo considerado as regiões com maiores índices de dor as regiões de pescoço $(0.22 \mathrm{p}$-valor $=$ $0.14)$, coluna (0.09 p-valor $=0.79)$, mãos (0.03 p-valor $=0.79)$, punhos $(0.06 \mathrm{p}$-valor $=0.64)$, membros inferiores $(0.10 p$-valor $=0.48)$, pé e tornozelos $(-0.02$ $p$-valor $=0.89)$. As correlações positivas são encontradas quando relacionadas à presença de dor no sexo feminino (0.03 e $p$ valor $=0.83$ ), há hipóteses condizentes e que também se encontram em outros artigos, referem se ao fato de que a dupla jornada de trabalho da mulher, maior repressão gerando medo, tensão e estresse, além de as trabalhadoras geralmente serem responsáveis por trabalhos mais minuciosos e apresentarem uma proporção dos tipos de fibras musculares diferentes dos homens. 
Ao relacionar dor e idade, não foi observada uma relação significativa, assim como na literatura não foi encontrada qualquer relação entre a idade e a propensão para desenvolver dores ocupacionais, carece de melhor pesquisa nesse aspecto. Temos uma relação positiva quando relacionada ao tempo na função, visto que a realização de movimentos de caráter repetitivos predispõe a patologias musculoesqueléticas como lesão por esforço repetitivo (LER) ou distúrbios osteomusculares relacionados ao trabalho (DORT).

A jornada do profissional da enfermagem que realiza o transporte de pacientes e de materiais no Centro Cirúrgico é dotada de intercorrências de acordo com as observações, desde a saída do paciente do centro cirúrgico até a sala de recuperação pós anestésica. Posteriormente, após o paciente restabelecer seus sinais vitais, deve retornar à enfermaria, esse retorno na maioria das vezes é realizado pelo enfermeiro circulante.

São episódios como a falta de recursos humanos, problemas relacionados à manutenção de equipamentos e falhas na comunicação que precisam ser analisados, uma vez que, comprometem a segurança da assistência e podem levar à ocorrência de um evento adverso. E ainda quando combinada com um risco ergonômico e ou ocupacional é capaz de levar a algum acidente no próprio ambiente de trabalho ${ }^{6}$.

Para Novaretti MCZ, Santos E de V, Quitério LM, e Daud-Gallotti RM ${ }^{22}$. as circunstâncias notificáveis mais prevalentes são condições de trabalho da equipe de enfermagem, em grande parte marcada pela inadequação do dimensionamento pessoal e sobrecarga de trabalho, esses fatores comprometem a saúde do profissional e interferem na qualidade do cuidado proporcionado ao paciente.

Uma das formas de prevenir o estresse e manter a qualidade de vida é a prática de atividade física regular; a ginástica laboral realizada no ambiente de trabalho, é um importante fator promocional da saúde, gerando benefícios orgânicos, emocional e social.
No presente estudo, sobre a oferta de ginástica Trabalho, 69,1\% disseram que o hospital oferta a ginástica laboral, mas apenas 35,7\% participam ${ }^{17}$. Kleinubing 25 , enfatiza que o conhecer dos fatores estressores pode auxiliar as instituições e os profissionais a repensar seu processo de trabalho para tornar o cotidiano mais produtivo e menos desgastante, o que refletirá na qualidade da assistência prestada.

\section{Conclusão}

A presença de dor foi maior em mulheres, independentemente da idade e, foi influenciada pelo setor ocupado e a função de enfermeiro, auxiliar ou técnico. Com relação aos sinais e sintomas foram maiores as queixas quando frequentemente agravados, na cabeça, na coluna, nos braços. Além disso a ocorrência de desânimo, ansiedade ou irritabilidade e quando relacionados a eventualidades, foram na cabeça, no peito, na coluna, no estômago e nos braços.

A justificativa para a repetição destas queixas está relacionada ao sistema musculoesquelético e psicológico, já que estes desempenham maior papel durante o desempenho do trabalho da enfermagem. Desse modo, tornam-se necessárias a adoção de medidas preventivas que visem a melhoria do estado de saúde prevenindo complicações musculoesqueléticas no momento do trabalho.

Ademais, a melhoria e adequação da inserção do profissional fisioterapeuta neste ambiente de trabalho deve ser pensada para melhor realização de medidas preventivas, e também na prescrição correta de exercícios, de acordo com as características únicas de cada indivíduo, em frente às demandas propostas diariamente seu ambiente de trabalho. 


\section{Contribuições dos autores}

Quevedo VS participou da coleta de dados qualitativos da pesquisa, interpretação dos dados e redação do artigo científico. Miranda FC participou da correção e da coleta de dados quantitativos da pesquisa. Chomem P participou da correção, redação e coleta de dados quantitativos. Bayerl R participou da coleta de dados da pesquisa, interpretação dos dados e resultados. Legey ALC participou da busca e análise estatística dos dados da pesquisa interpretação dos resultados. Motter AA participou da concepção, delineamento, orientação e correção do artigo.

\section{Conflitos de interesses}

Nenhum conflito financeiro, legal ou político envolvendo terceiros (governo, empresas e fundações privadas, etc.) foi declarado para nenhum aspecto do trabalho submetido (incluindo, mas não se limitando a subvenções e financiamentos, participação em conselho consultivo, desenho de estudo, preparação de manuscrito, análise estatística, etc.).

\section{Referências}

1. Castro MM, Oliveira SS. Avaliação do trabalho na Atenção Primária à Saúde do município do Rio de Janeiro: uma abordagem em saúde do trabalhador. 2017; 41(Especial):152-164. doi: 10.1590/0103-110420175213

2. Santos EC, Andrade RD, Lopes SGR, Valgas C. Prevalência de dor musculoesquelética em profissionais de enfermagem que atuam na ortopedia. Rev. Dor. 2017;18(4):298-306. doi: 10.5935/1806$\underline{0013.20170119}$

3. Vidor CR, Mahmud MA, Farias LF, Silva CA, Ferrari JN, Comel JC et al. Prevalência de dor osteomuscular em profissionais de enfermagem de equipes de cirurgia em um hospital universitário. Acta Fisiátrica. 2014;21(1):6-10. doi: 10.5935/0104-7795.20140002

4. Genç A, Kahraman T, Göz E. The prevalence differences of musculoskeletal problems and related physical workload among hospital staff. J Back Musculoskel Rehabil. 2016;29(3):541-7. doi: 10.3233/BMR-160655

5. Freimann T, Coggon D, Merisalu E, Animägi L, Pääsuke M. Risk factors for musculoskeletal pain amongst nurses in Estonia: a cross-sectional study. BMC Musculoskel Disord. 2013;14:334. doi: 10.1186/1471-2474-14-334

6. Rossete, Celso Augusto. Segurança e higiene do trabalho. São Paulo: Pearson Education do Brasil; 2014.
7. Gerr F, Fethke NB, Merlino L, Anton D, Rosecrance J, Jones MP et al. A prospective study of musculoskeletal outcomes among manufacturing workers: I. effects of physical risk factors. Hum Factors. 2014;56(1):112-30. doi: 10.1177/0018720813491114

8. Cordeiro SBM, Araújo TM, Almeida MMG, Santos KOB. Características sociodemográficas e condições de saúde da população urbana de Feira de Santana, Bahia: análise de diferenciais de gênero. Revista Baiana de Saúde Pública, 2011, 35(1), 9-27.

9. Levesque JF, Mukherjee S, Grimard D, Boivin A, Mishra S. Measuring the prevalence of chronic diseases using population surveys by pooling self-reported symptoms, diagnosis and treatments: results from the World Health Survey of 2003 for South Asia. Int J Public Health. 2013;58(3):435-47. doi: $10.1007 /$ s00038-013-0446-5

10. Castro R, Elias FTS. Involvement of patients of healthcare systems in Health Technology Assessment (HTA): a narrative review of international strategies. Interface (Botucatu). 2018; 22(64):97-108. doi: 10.1590/1807-57622016.0549

11. Lima AC, Magnago TS, Prochnow A, Ceron MD, Schardong $\mathrm{AC}$, Scalcon $\mathrm{CD}$. Fatores associados à dor musculoesquelética em trabalhadores de enfermagem hospitalar. Rev Enferm UERJ. 2014;22(4):526-32.

12. Lida I, Buarque LIA. Ergonomia: projeto e produção. 3. ed. São Paulo: Edgard Blucher Ltda; 2016.

13. Gómez-Bull KG, Hernández-Arellano Jl, Ibarra-mejía, G. A proposed methodology for task analysis in ergonomic evaluations. Procedia Manufacturing. 2015;4756-4760. doi: 10.1016/j.promfg.2015.07.573

14. Leite Es, Silva EN, Santos J. Educação continuada na central de material e esterilização: significados e dificuldades enfrentadas pela enfermagem. Rev SOBECC. 2011;16(4):31-9.

15. A Associação Americana de Enfermagem American Nurses Association. What Is nursing? Estados Unidos da América. [Internet]. 2016. [acesso em 17 outubro de 2018]. Disponível em: http://www.scielo.br/pdf/rlae/v25/pt_0104-1169-rlae-25-e2913.pdf

16. Steyrer J, Schiffinger M, Huber C, Valentin A, Strunk G. Attitude is everything? The Impact of workload, safety climate, and safety tools on medical eroors: a study of intensive care units. Health Care Manage Rev. 2013;38(4):306-316. doi: 10.1097/ HMR.0b013e318272935a

17. Araújo LC, Oliveira MRf, Pereira RCC, Félix TA, Dias RA, Dias MSA. Ginástica laboral em ambiente de emergência: relato de experiência no pet-saúde "Redes de Atenção". SANARE - Revista de Políticas Públicas. 2015;14(1):87-92. 
18. Reed LF, Battistutta D, Young J, Newman B. Prevalence and risk factors for foot and ankle musculoskeletal disorders experienced by nurses. BMC Musculoskelet Disord. 2014;15:196. doi: $\underline{10.1186 / 1471-2474-15-196}$

19. Maciel Rhmo, Santos Jbf, Rodrigues RI. Condições de trabalho dos trabalhadores da saúde: um enfoque sobre os técnicos e auxiliares de nível médio. Rev Bras Saúde Ocup.2015;40(131):7587. doi: 10.1590/0303-7657000078613

20. Nauderer, Taís Maria And Lima, Maria Alice Dias Da Silva. Imagem da enfermeira: revisão da literatura. Rev Bras Enferm. 2005;58(1):74-77. doi: 10.1590/S0034-71672005000100014

21. Gonçalves La, Andolhe R, Oliveira Em De, Barbosa RI, Faro Acm, Gallotti Rmd, et al. Alocação da equipe de enfermagem e ocorrência de eventos adversos/incidentes em unidade de terapia intensiva. Rev Esc Enferm. 2012;46 (Esp):71-7. doi:10.1590/S0080$\underline{62342012000700011}$

22. Novaretti MCZ, Santos EV, Quitério LM, Daud-Gallotti RM. Sobrecarga de trabalho da enfermagem e incidentes e eventos adversos em pacientes internados em UTI. Rev Bras Enferm. 2014; ;67(5):692-9. doi: 10.1590/0034-7167.2014670504

23. Sousa PTM, Sousa ARR, Pacheco ES, Sousa GTM.

Comportamento sedentário entre profissionais da Estratégia de Saúde da Família. Rev Enferm UFPI. 2017;6(3):24-9. doi: 10.26694/ reufpi.v6i3.5764

24. Matsudo S, Araujo T, Matsudo V, Andrade D, Andrade E, Oliveira $C$ et al. Questionário Internacional de atividade física (IPAQ): Estudo de Validade e reprodutibilidade no Brasil. Revista Brasileira de atividade física e saúde, 2001;6(2): p.5-12. doi:

10.12820/rbafs.v.6n2p5-18

25. Kleinubing RE, Goulart CT, Silva RM, Umann J, Guido LA. Estresse E Coping Em Enfermeiros De Terapia Intensiva Adulto E Cardiológica Stress And Coping In Nurses Of Adult And Cardiological Intensive Care Estrés Y Coping En Enfermeros De Cuidados Intensivos Adulto Y Cardiológico. Rev Enferm UFSM. 2013;3(2):335-344. doi: 10.5902/217976928924 